\title{
MANEJO DE LA QUEMA DE PASTIZALES DE SABANA INUNDABLE: UNA MIRADA DEL PUEBLO ORIGINARIO SÁLIVA EN COLOMBIA
}

\author{
THE MANAGEMENT OF THE BURNING OF FLOODED SAVANNA \\ GRASSLANDS: A LOOK FROM THE NATIVE SÁLIVA PEOPLE IN COLOMBIA
}

\author{
Alejandro Huertas Herrera ${ }^{1}$, Brigitte Luis Guillermo Baptiste Ballera ${ }^{2}$, Mónica Toro Manríquez ${ }^{1}$ y \\ Hugoberto Huertas Ramírez $z^{3}$
}

\begin{abstract}
El conocimiento histórico que se tiene de las quemas por parte de los pueblos originarios contribuye a entender el papel que cumple el fuego en ecosistemas naturales tropicales y templados. El objetivo de este trabajo fue describir el manejo y la importancia de la quema para el pueblo originario Sáliva en el ecosistema de sabana inundable de Colombia. A través de entrevistas semiestructuradas, cartografía social y calendarios anuales se obtuvieron las apreciaciones que tienen los Sáliva del fuego en pastizales nativos. La percepción generalizada de que las quemas son perjudiciales se ha insertado en su imaginario, pero se conserva el concepto del fuego como un factor determinante que afecta la sucesión vegetal y su uso mantiene el paisaje de sabana. Antes de ser aculturizados por los jesuitas en costumbres, religión y ganadería bovina, los Sáliva aplicaban fuego para facilitar la caza y los desplazamientos, pero su sometimiento al manejo del ganado cambió la concepción de su aplicación. Se concluyó que en la actualidad los Sáliva no aplican fuego indiscriminadamente, sino que hacen un manejo estratégico con fines agropecuarios y de prevención de incendios y rescatan saberes tradicionales del conocimiento ecosistémico.
\end{abstract}

Palabras claves: fuego, paisaje, ganadería, aculturación, Orinoquía, Orocué.

The historical knowledge about the management of burnings by native peoples allows us to understand the role played by fire in natural tropical and temperate ecosystems. The objective of this work was to describe the management and importance of burning for the native Sáliva people in the flooded savanna ecosystem of Colombia. The assessment that the Sáliva have of fire in native grasslands was obtained through semi-structured interviews, social cartography and annual calendars. The generalized opinion that burnings are harmful is firmly established in their imaginary, but the concept of fire remains as a determining factor affecting plant succession and its use maintains the savanna landscape. Before being acculturized by the Jesuits in customs, religion and cattle rearing, the Sáliva applied fire to facilitate hunting and movement, but their subjection to cattle rearing changed the conception of its application. It is concluded that, at present, the Sáliva do not apply fire indiscriminately; instead, they manage it strategically for agricultural purposes and also rescue traditional knowledge of the ecosystem.

Keywords: Fire, landscape, cattle rearing, acculturation, Orinoquía, Orocué.

Los Sáliva son un pueblo originario de los llanos venezolanos del Estado Apure (Piraban 2011). Eran nómadas expertos en pesca y caza, por lo que su territorio abarcó vastas extensiones de los llanos colombo-venezolanos (Morey 1972), que vieron la necesidad de aplicar fuego a las extensas sabanas tropicales para facilitar el desplazamiento a pie, la caza y prevenir ataque de fieras; no existían limitaciones de propiedad que restringiera la quema, que se apagaba por sí sola. La primera gran transformación en esta práctica ancestral se produjo con la colonización europea de los llanos orinocences de Colombia por obra de los jesuitas cuando fundaron la hacienda ganadera Caribabare en territorios de los actuales

\footnotetext{
${ }^{1}$ Centro Austral de Investigaciones Científicas, Consejo Nacional de Investigaciones Científicas y Técnicas, Ushuaia, Tierra del Fuego (9410), Argentina. ahuertasherrera@cadic-conicet.gob.ar; monicatm@cadic-conicet.gob.ar

2 Instituto de Investigación de Recursos Biológicos Alexander von Humboldt, Bogotá D.C., Colombia. brigittebaptiste@humboldt.org.co

${ }^{3}$ Fundación Horizonte Verde, Cumaral, Meta, Colombia. hugobertohuertas@gmail.com
} 
departamentos de Casanare y Arauca; labor análoga al resto de países de Latinoamérica, p.ej., Paraguay (Klaiber 2007); la concentración de Sálivas en esta y posteriores haciendas, más la huida hacia territorios selváticos redujeron las quemas anuales de sabana, pero algunos relatos refieren grandes e incontrolables incendios espontáneos (Huertas Herrera 2006). Con la culturización ganadera se reactivó la aplicación del fuego con el propósito esencial de suministrar retoño de gramíneas fibrosas y secundariamente para facilitar la recolección de ganado. Luego de la expulsión de los jesuitas en 1769, tanto el ganado, como los Sálivas y mestizos quedaron al garete; de esta manera surgieron pequeñas haciendas de su propiedad, donde continuaron aplicando lo aprendido con los jesuitas, con la finalidad de proteger de incendios sus viviendas en palma y madera (Figura 1) y facilitar la cacería (Huertas Ramírez y Huertas Herrera 2015). Desde que se convirtieron en sedentarios cultivan yuca amarga (Manhiot sp.), de donde obtienen el casabe y el mañoco, alimentos indispensables en su dieta. En la actualidad son ganaderos, poseen bovinos y equinos en territorios denominados resguardos indígenas; en Colombia se encuentran principalmente en los resguardos El Consejo, El Duya, El Medano, El Saladillo, El Suspiro, Macucuana, Paravare, y San Juanito, localizados en las sabanas inundables del Municipio de Orocué, Departamento de Casanare (Figura 2), asentándose a orillas de ríos, bosques de galería y en sabanas.

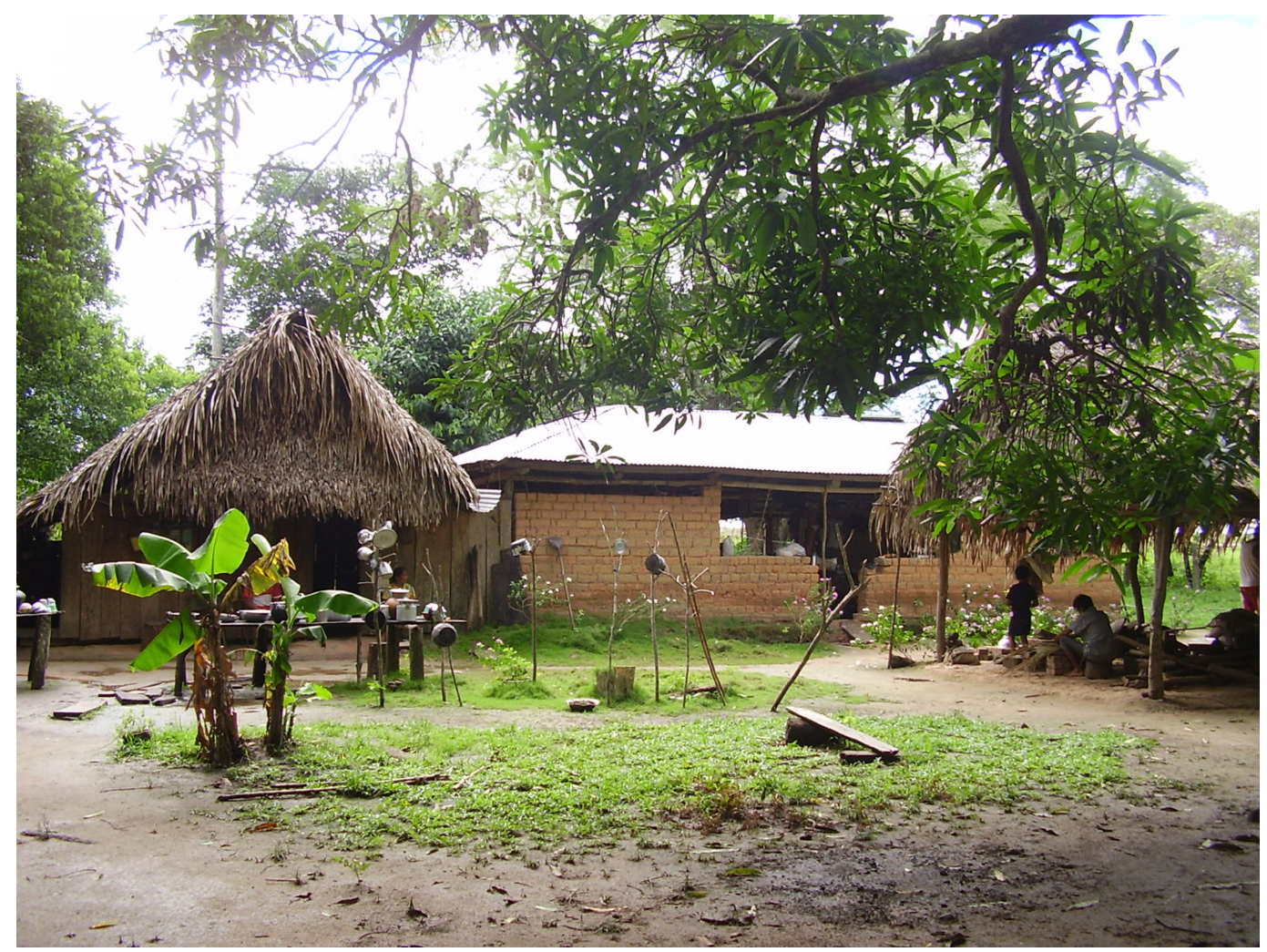

Figura 1. Vivienda actual del pueblo originario Sáliva.

Current housing of the Sáliva. 


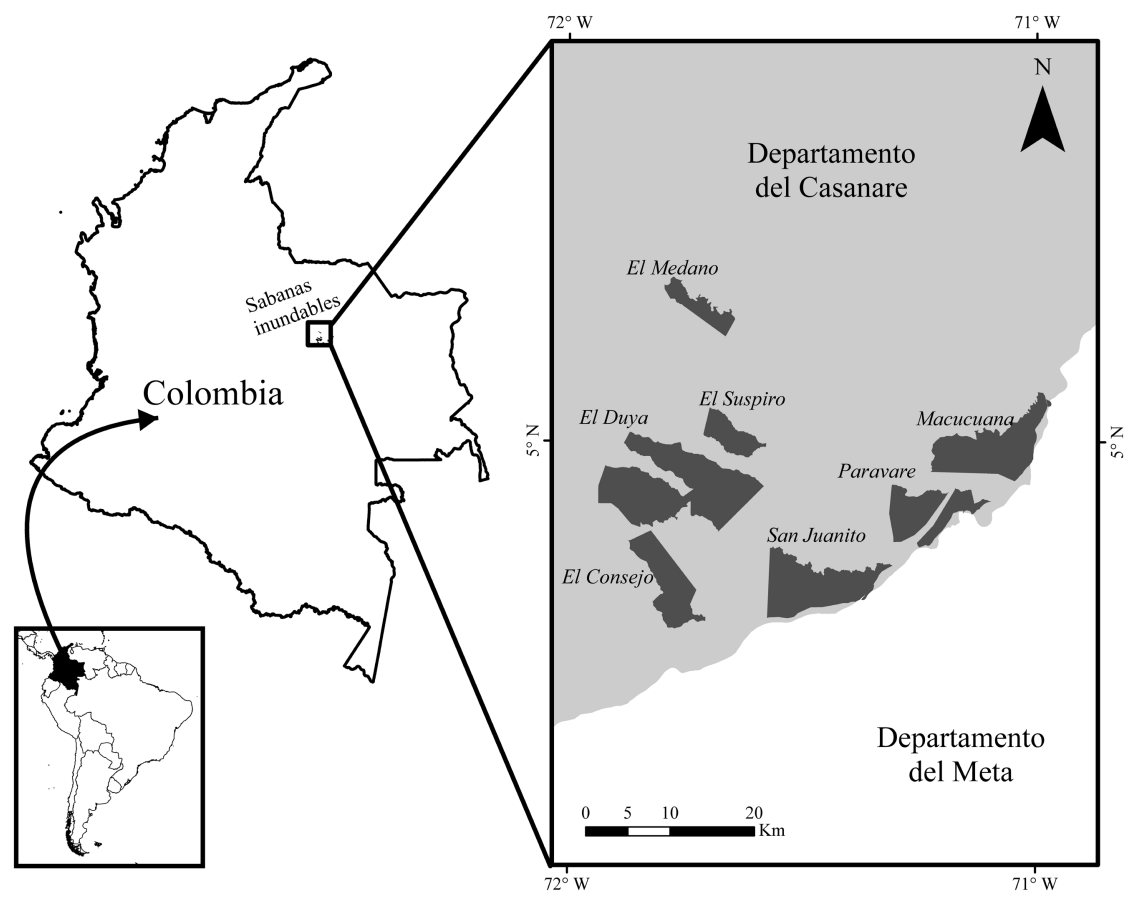

Figura 2. Mapa de localización de los actuales territorios Sáliva en el Departamento de Casanare. Location map of the current Sáliva territories in the Department of Casanare.

Las sabanas son el ecosistema predominante del paisaje y se caracterizan por tener una topografía plana cóncava, cuyas zonas que no se inundan durante época de lluvias, se conocen como bancos, y las zonas inundadas como bajos y esteros (humedales) (Figura 3). En los meses de lluvias (abril-noviembre) las zonas cóncavas mantienen espejos de agua por acción directa de la precipitación y/o el desborde de ríos y caños. Estas características del relieve son determinantes para el uso de su territorio, pues las actividades agropecuarias y disponibilidad forrajera dependen de la dinámica del agua cosechada de manera natural.

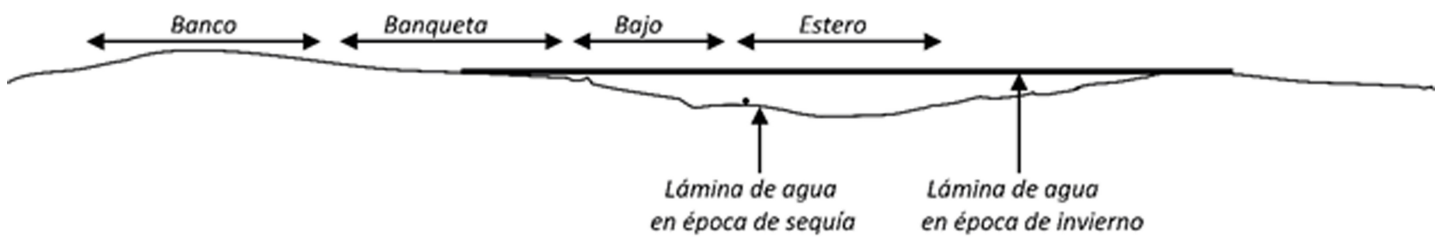

Figura 3. Fisiografía de la sabana inundable.

Physiography of the flooded savanna. 
El aprovechamiento de las sabanas requiere de perturbaciones, como el fuego, para generar y sostener la coexistencia de árboles y pastos mediante el desarrollo de heterogeneidad (Mistry et al. 2005; Rodríguez 2004). En los llanos orinoquences de Colombia y Venezuela ha sido una práctica ancestral por más de 10.000 años, la cual limita la sucesión vegetal y por consiguiente la presencia de formaciones boscosas, y mantiene el paisaje de sabana como lo conocemos (Huertas Herrera 2006; Rodríguez 2007). Sin embargo, la creencia de que el fuego es intrínsecamente dañino para los ecosistemas tiende a restringirse en lugares donde es un factor ecológico determinante y parte de la idiosincrasia de los pueblos originarios (Laris 2002; Lewis 1989; Rodríguez 2007), pues la expansión de la frontera agrícola, pecuaria y forestal de las últimas décadas ha modificado en los pobladores el patrón del uso del fuego (McGregor et al. 2010; Mistry et al. 2005) por parcelamiento de predios, introducción de pasturas que no se queman, cultivos forestales y por la emisión de gases de efecto invernadero que inciden en el cambio climático, entre otros (Lewis 1989; Mistry et al. 2005). En consecuencia, el objetivo de este trabajo fue describir el manejo y la importancia de la quema para el pueblo originario Sáliva en el ecosistema de sabana inundable de Colombia, correspondiente al municipio de Orocué.

Utilizamos dos marcos teóricos para comprender las connotaciones del uso del fuego en comunidades Sáliva que incluyen la etnografía para comprender el contexto cultural de los grupos focales y la interpretación de sus experiencias para conceptualizar las interrelaciones entre las personas, los organismos y su entorno (Aedo 2004; Jones y Smith 2017), y un marco de conservación de la naturaleza (Fischer et al. 2014; Lindenmayer et al. 2008) para comprender cómo el fuego está fuertemente vinculado a los sistemas socioecológicos Sáliva y el enorme potencial que su conocimiento ofrece al abordaje de problemáticas ambientales contemporáneas en ecosistemas naturales tropicales y templados (p.ej., desarrollo sustentable y manejo del territorio), ambos sustentados de entrevistas y experiencias empíricas para analizar la visión de la naturaleza en las personas y las comunidades (Jones y Smith 2017).

\section{Métodos}

\section{Recopilación de datos}

La investigación se centra en información etnográfica obtenida en los resguardos El Duya, Paravare y San Juanito. Estos fueron seleccionados aleatoriamente entre los ocho resguardos del Municipio de Orocué. Se realizó una fase de campo preliminar durante el periodo de verano de 2005 (enero y febrero) con el propósito de reconocer el área de estudió, entablar un diálogo preliminar con los líderes de las comunidades (presentarse formalmente), familiarizarse con el lenguaje local (p.ej., los nombres vernáculos de las especies) y realizar entrevistas preliminares (10 personas) de un enfoque adaptativo en las que se fueron ajustando palabras y conceptos relevantes de la zona para evitar tecnicismos y conocer el sentido que le atribuyen los indígenas a distintas prácticas o términos en español de la vida cotidiana (p.ej., incendio $=$ candelazos; hacienda $=$ hato citadino $=\mathrm{el}$ blanco). Esta fase permitió dilucidar un marco teórico con preguntas e información importante respecto a las comunidades estudiadas (p.ej., costumbres) y el uso del fuego. Posteriormente, se visitaron estas comunidades durante enero y abril de 2006, donde se entrevistó a 30 personas de ambos géneros, diez por cada resguardo (5 mujeres y 5 hombres), cuyo rango de edad varió de 25 a 75 años. Los entrevistados se dedicaban a la agricultura (p.ej., empírica en conuco o huerta casera para autoconsumo) y pesca de subsistencia y a la ganadería bovina (ganado cebú, Bos indicus). Se diseñó un formato de entrevista semiestructurada que contó con 24 preguntas, cuyas respuestas no superaron 45 minutos de duración por persona, grabadas digitalmente y transcritas para el registro y consecuente análisis de la información. Todos los entrevistados hablaban español, y se entrevistó uno a uno en sus hogares; para garantizar que la información fuera lo más imparcial posible se evitó la presencia de otras personas durante las entrevistas. Cabe mencionar que se memorizaron las 24 preguntas con su correspondiente orden con el fin de no intimidar a los entrevistados, y se incorporaron algunas palabras de la lengua Sáliva para generar un diálogo natural y ameno (p.ej., sabana $=k \tilde{a} d e$; fuego $=$ oosa , gracias = bae kelegiaja). Además, se elaboraron calendarios anuales y mapas con la cartografía social de cada territorio para una mejor interpretación de la información recopilada. Todas las actividades se realizaron con el consentimiento de los líderes de las comunidades.

\section{Análisis de datos}

Se transcribieron e interpretaron las entrevistas considerando el género y rango de edad de las personas. Con base en Miles y Huberman (1994), se construyó una matriz de datos en la que se categorizaron las respuestas de los entrevistados en función de tres temas principales: el conocimiento del manejo del fuego como configurador del paisaje, las prácticas agrícolas y ecología de las especies de pastizales de sabana inundable. Para construir la matriz se relacionaron conceptos y palabras relevantes al marco teórico del trabajo (p.ej., uso del fuego, especies de plantas, 
manejo animal) y se consideró el número de veces que emergieron las respuestas de los entrevistados. Esta información fue integrada en análisis con los calendarios anuales y mapas con la cartografía social, considerando además otros temas que no correspondían a las entrevistas (p.ej., sitios sagrados, lugares para la fauna, conflictos por reglamentación y uso del fuego). Los calendarios y mapas se realizaron a través de talleres con la comunidad y recorridos de verificación en los tres territorios.

\section{Resultados}

\section{Connotaciones del uso del fuego}

Los resultados indican que el manejo del fuego en sabana es una práctica llevada a cabo casi en su totalidad por los varones (90,2\% de los entrevistados), realizada "a pie" y que rara vez incluye la monta de caballo (Equus ferus). El 72,5\% de los entrevistados manifestó que en ausencia de las quemas se acumula material leñoso en la sabana con potencial altamente inflamable de forma natural (p.ej., rayos) o por acción antrópica. El $35,0 \%$ de las mujeres manifestó que el uso de la quema es para la seguridad del grupo familiar, pero aplicada de manera controlada del material leñoso acumulado cerca de sus viviendas hechas con madera y palma. En los tres resguardos, el 86,2\% de los entrevistados coincide en que el ecosistema de sabana, en ausencia de pastoreo bovino posterior al fuego, se recupera rápidamente (es resiliente). Destacaron, además, que el fuego afecta de forma diferencial la vegetación y a la fauna en las áreas de bosque, principalmente en los bosques de galería, reconociéndolos como un sistema natural más frágil al fuego con respecto a la sabana.

\section{Épocas, zonas y control de la quema}

Las entrevistas, cartografía social y el calendario anual indican que, por las características fisiográficas de las sabanas inundables, las quemas para la alimentación del ganado son focalizadas y anuales y se realizan en períodos de lluvia y de sequía. En época de lluvias queman zonas de banco o "zonas no inundables" y en época de sequía algunas partes de los bajos o "zonas inundables". Cuando en abril llegan las lluvias se acumula paulatinamente agua en los bajos y esteros, lo que ocasiona que el pastoreo bovino predomine en las áreas de banco, por lo que los Sáliva tienden a manejar el fuego de acuerdo a la dinámica de lluvias e inundación; es así que las quemas se realizan en varios períodos del año, pero en diferentes áreas.

Las quemas son aconsejables, pero en buena época, porque si uno va a quemar una sabana que esté algo pantanosa (que esté húmeda) y que no esté bien seca la raíz del pasto, al meterle candela esa agua va a calentar el pasto y lo daña, el pasto se muere. Entonces hay que procurar que la sabana esté seca. Ojalá que estuviera uno de buenas, que quemó y le cayó un aguacero después. Entonces las quemas son necesarias, pero uno debe tener conocimiento. Aunque hay unos que tienen una forma de quemar, otros otra. Algunos dañan la sabana; unos le meten candela y en lugar de salir pasto sale es maleza (hombre entrevistado de 64 años).

En diciembre, cuando inicia la sequía, va desapareciendo el espejo de agua del bajo y se aplica fuego en el área periférica al banco; en abril cuando inicia el período de lluvias, se queman algunas áreas de banco para que los bovinos dispongan de rebrote tierno y nutritivo. En el mes de agosto disminuyen los días lluviosos, período conocido como "veranillo", se queman partes de los bancos que no se quemaron en abril. En general, los períodos de sequía son particularmente peligrosos para realizar y controlar quemas; cuando es incontrolable se llama "candelazo" (incendios a gran escala $>100$ hectáreas e intensos). Hoy en día no son frecuentes por el menor tamaño de las haciendas ganaderas y cultivos agrícolas delimitados con alambre y por estar los Sáliva restringidos en resguardos. Los candelazos eran provocados para limpiar grandes extensiones de sabana, lo cual era una forma de regular el paisaje, y para suministrar retoño de pastizal leñoso. Para evitar candelazos, se tiene en cuenta la hora del día, la cantidad de material vegetal seco, la fuerza y dirección del viento. Si es grande el área a quemar prefieren hacerlo temprano en la mañana donde los vientos son fuertes y la temperatura es alta; si es un área pequeña prefieren al atardecer o durante la noche cuando la temperatura baja y el viento es menos intenso; de este modo no avanza rápidamente.

Por ahí a las 3 de la tarde es bueno quemar, y de ahí en adelante, porque ya llega la noche y fresquea (baja la temperatura), entonces las candelas se apagan y no queman tanto. Usted mete una candela a las 8 de la mañana, y eso pasa de su sabana y sigue para la otra y se forman candelazos. Eso toca irle quemando pedazo por pedazo, porque si se quema todo al tiempo hay retoño que se pierde y ahí sufre el ganado (hombre entrevistado de 70 años).

En ambos escenarios un factor determinante es el material vegetal acumulado; si es escaso no representa mayor riesgo, pero si se ha acumulado por 
varios años se extrema la vigilancia para evitar daños o perjuicios en el interior del resguardo o al vecindario. Entre las estrategias de control más utilizadas están los "guarda rayas" y los "callejones"; los guarda rayas son pequeñas quemas longitudinales preliminares que cortan el avance del fuego hasta el área deseada; se usan para evitar traspasos a otros predios o territorios; los callejones son márgenes entre 1 a $2 \mathrm{~m}$ de ancho, limpios de pasto, especialmente a lo largo de las cercas, utilizando herramientas manuales o máquinas. Para apagar una quema se usan ramas o se echa tierra sobre el fuego.

\section{Conocimiento del fuego sobre las especies y el ecosistema}

El conocimiento local de la respuesta del ecosistema a la quema corresponde a la capacidad de rebrote de la vegetación, siendo las gramíneas las primeras en brotar; la intensidad y duración del fuego, como también su frecuencia y tiempo de ocurrencia, tiene un efecto diferencial en la vegetación determinado por la capacidad de rebrote y germinación de cada especie. Igualmente, manifestaron que en ausencia de fuego, y dependiendo del tipo de sabana, crece el bosque nativo y la fauna encuentra mayor refugio.

Cuando no se quema la sabana el pasto está muy grande y seco. Se empiezan a meter bichos por ahí como el güio (Eunectes murinus) y el cachicamo (Dasypus sabanicola). También anda el oso (Myrmecophaga tridactyla) y el morrocoy (Chelonoidis carbonaria), también la cuatro narices (Bothrops atrox), pero esa sí que es muy peligrosa (mujer entrevistada de 20 años).

No obstante, el conocimiento sobre las especies vegetales es restringido y hay personas dentro de las comunidades que tienen saberes más profundos de los efectos del fuego y el uso que tienen las plantas. Según el conocimiento de los Sáliva, el manejo que se dé al fuego en el ecosistema estimula el crecimiento o no de ciertas plantas; es así que un buen manejo del banco favorece las gramíneas guaratára (Axonopus purpusii) y gramas (Paspalum sp.), que son bien apetecidas por los bovinos sin necesidad de quemar; la quema mejora notoriamente el consumo por los bovinos de gramíneas muy lignificadas, como la saeta (Trachypogon vestitus); en la transición banco/bajo estimula el aprovechamiento de rabo de vaca (Andropogon bicornis), rabo de mula (Leptocoryphium lanatum) y rabo de gato (Andropogon leucostachyus). Sin embargo, las quemas en época de sequía en zonas cercanas a los humedales presentan el riesgo de afectar gramíneas que no lo requieren, como la lambedora (Leersia hexandra). Asimismo, varias plantas son empleadas por la comunidad para uso medicinal. Por ejemplo, la hierba paloma (Euphorbiahy pericifolia) y el mastranto (Hyptis sp.) son utilizados como antisépticos y el trompito (Solanum sp.) como diurético.

\section{Apreciaciones negativas del fuego}

Las apreciaciones negativas de las quemas se focalizan en los daños y perjuicios de bienes propios o ajenos, seguidos por la degradación del ecosistema que se refleja en el desplazamiento de la fauna y el cambio de la calidad del agua por las cenizas que ocasionan "embarbascamiento" y, por ende, mortandad de peces; algunos de ellos de importancia en su dieta como la guabina (Hoplias malabaricus), el curito (Hoplosternum litoralle) y el pavón (Cichla orinocensis). También se pueden afectar ecosistemas muy importantes para la comunidad, como los morichales (Mauritia flexuosa) o las matas de monte (bosques de galería), de donde extraen materiales y conservan especies que les son emblemáticas (p.ej., inspiración para canciones y relatos); por ejemplo, la corocora (Eudocimus ruber), la guacamaya (Ara macao), el loro real (Amazona ochrocephala), la maracana (Orthopsittaca monilata), el tigre o jaguar (Panthera onca) o el ocelote o tigrillo (Leopardus pardalis). A su vez, declararon efectos negativos sobre la fertilidad del suelo y menor vigorosidad de plantas que se asocian con la disminución del descanso necesario de los pastizales de sabanas, pero estas consideraciones no han tenido soporte investigativo. La reconfiguración de su territorio y la aplicación del fuego están ligados a la inclusión de nuevas apuestas productivas dentro de los resguardos.

\section{Discusión}

\section{Connotaciones del uso del fuego}

Como en muchas regiones con sabanas tropicales, el manejo del fuego es una práctica frecuente realizada principalmente para promover el rebrote fresco de pastizales fibrosos para la alimentación de bovinos $y$, en algunos casos, para el control de incendios al no permitir la acumulación de material vegetal seco (Rippstein et al. 2001; Rodríguez et al. 2016).

Nuestros resultados muestran que las quemas de sabana son utilizadas para uso pecuario (alimentación de bovinos), control de grandes incendios al impedir que se acumule el material vegetal, y regular la biodiversidad del ecosistema (p.ej., disminuir plagas, ahuyentar especies peligrosas). El uso del fuego en comunidades indígenas es para muchos, 
como los adultos mayores, una parte integral de su cultura, profundamente enraizada en sus prácticas tradicionales, por lo tanto, cualquier intento de eliminar o restringir el uso del fuego inevitablemente se verá como una amenaza a su identidad cultural y su sentido de propiedad sobre sus tierras, y es probable que encuentre resistencia (Rodríguez 2007). Esto también concuerda con observaciones de Mistry et al. (2005), quienes resaltan que el conocimiento del uso del fuego de Krahô se está perdiendo debido al crecimiento de la frontera agrícola (soya y ganadería), desatando además inevitables conflictos socioambientales inherentes al uso del fuego en el territorio.

Los conflictos por el uso del fuego por pueblos indígenas han sido ampliamente documentados en el mundo, particularmente en parques nacionales (Fairhead y Leach 1996). Estudios previos han encontrado mayor aceptación del uso del fuego en ecosistemas de sabanas por parte de pueblos indígenas, aunque esta sigue siendo una práctica local altamente controversial (Rodríguez et al. 2016). En Australia y África Occidental, demuestran que los indígenas utilizan la quema de sabana como herramienta de manejo de la tierra para prevenir grandes incendios forestales (Rodríguez 2004); en África Occidental y Australia se ha determinado que el uso del fuego por prácticas indígenas en lugar de contribuir con la sabanización es una herramienta fundamental para el manejo de mosaicos dinámicos de sabana y bosquessabana (Mbow et al. 2000). Es preocupante que las nuevas generaciones Sáliva desconozcan saberes ancestrales sobre aplicación controlada del fuego y le estén dando más relevancia a lo que consideran en la actualidad más rentable económicamente, como es la ganadería, sin mayores consideraciones de las afectaciones ambientales, lo que se podría contrarrestar con capacitaciones que equilibren la estrategia producción/conservación y que valoren fuertemente su lengua e identidad cultural. Por ejemplo, conceptos derivados de la denominada revolución verde en Estados Unidos, como "pastos mejorados" (especies introducidas) o "malezas" (especies nativas), fueron frecuentemente mencionados en las entrevistas, lo que denota un cambio generalizado en el imaginario Sáliva y eventuales problemáticas sobre el uso, manejo y conservación de los recursos naturales de su territorio, pues tienden a percibir que lo exótico es inherentemente "bueno" y lo nativo es "malo"; p.ej., en la cartografía social se encontró que en los tres resguardos, principalmente en San Juanito y el Duya, los Sáliva han accedido al reemplazo de cientos de hectáreas de pasturas nativas de rica biodiversidad y productividad manejable con la aplicación del fuego o guadaña manual por pasturas sembradas con especies exóticas comerciales de alto costo de implantación, lo que implica un cambio a escala de paisaje de sabanas nativas por monocultivos.

\section{Épocas, zonas y control de la quema}

En nuestro estudio, los reguladores más importantes para el uso del fuego son: la fisiografía de la sabana (banco, baqueta, bajo y estero) y la época del año (verano = sequía $;$ invierno = lluvias), regulado en algunos sectores de la sabana en función de la productividad y composición de la vegetación útil para la alimentación animal (principalmente bovino); por ejemplo, la gramínea guaratára (Axonopus purpusii). Estudios similares en Mali (África), develan como distintos actores sociales rurales manejan el fuego anualmente, lo cual comienza en la estación seca para fragmentar el paisaje, con el objetivo de prevenir incendios posteriores que puedan dañar los recursos naturales (Laris 2002). El proceso de quemar gradualmente la vegetación más seca crea un mosaico estacional de parches de hábitat que aumenta el potencial del paisaje para una variedad de usos de la temporada seca, incluida la caza, la recolección de productos de sabana y el pastoreo (Laris 2002). Otros estudios también han llegado a la conclusión de que el uso del fuego es imprescindible en las comunidades de pueblos originarios de Venezuela, revelando que, contrariamente a la creencia convencional, el uso del fuego no es producto de ignorancia o negligencia (Rodríguez 2007), o sea, implica un manejo estacional y zonal del territorio.

\section{Conocimiento del fuego sobre las especies y el ecosistema}

Los Sáliva perciben que el manejo que hacen del fuego facilita el crecimiento de determinadas plantas y regula la fauna local, y esto se ha insertado en su identidad y reconocen al ecosistema de sabana como un ambiente resiliente al fuego. Captan más beneficios ecológicos con una "sabana limpia" (sin vegetación lignificada) sin el riesgo de que el ecosistema colapse. En contraste, en muchas regiones del mundo los incendios crean áreas con suelo desnudo o cubiertos con pastizales dominados por especies que imposibilitan la recuperación de la vegetación nativa, y desatan un desequilibrio ambiental (Whelan 1995), por ejemplo, la presión de herbívoros en zonas que han sido afectadas por incendios forestales (Iriarte 2002). Esto es análogo a lo que sucedió en los bosques patagónicos de Chile y Argentina entre los años 1936 y 1956; como respuesta al proceso de habilitación de terrenos fiscales para uso agropecuario se quemaron más de 4.000 .000 de hectáreas de bosques nativos (Quintanilla 2008). Sin embargo, en las sabanas de Colombia, el fuego se 
reconoce como una herramienta indispensable para el control del ecosistema y la producción pecuaria, pues mantiene la estructura y composición de los pastizales para la alimentación de la fauna local y bovinos, coadyuvada por la humedad del suelo durante nueve meses continuos. Huertas Ramírez (1977) comprobó que con la quema de forrajes lignificados surge un rebrote bien apetecido por el ganado y de mayor calidad nutricional, así como mejoras en la mineralización de los animales por el consumo de cenizas. Además, la resiliencia del ecosistema de sabana inundable dependerá de la frecuencia con que se queme un sitio y el sometimiento a pastoreo de bovinos (Huertas Herrera 2006).

En sabanas de Brasil, el uso del fuego por parte del pueblo originario Krahô brinda un funcionamiento integral (bosques-pasturas) de la sabana a través de las perturbaciones (Mistry et al. 2005). Además, el manejo de la quema no solo previene los daños por grandes incendios, sino que también aumenta la biodiversidad (Laris 2002). En Australia, el manejo de la quema se basa en un profundo conocimiento experiencial, perfeccionado durante generaciones de vida íntima con el paisaje (McGregor et al. 2010). Sin embargo, las actitudes eurocéntricas sobre el uso del fuego son completamente distintas a la de los pueblos originarios (Lewis 1989).

\section{Apreciaciones negativas del fuego}

La práctica actual de la quema se relaciona con la economía ganadera adoptada desde el siglo XVII con las primeras misiones evangelizadoras jesuitas en sus haciendas localizadas en los Departamentos de Arauca y Casanare, que al paso de los siglos el ganadero llanero heredó las costumbres del cuidado de ganado (Huertas Ramírez y Huertas Herrera 2015). En la actualidad, los Sáliva tienen un manejo de la quema análoga a la del hombre llanero o "blanco" y cuentan con bovinos que pastorean dentro de los resguardos, la mayoría adquiridos por compensaciones o proyectos productivos de compañías establecidas en sus territorios, principalmente del sector hidrocarburos (Cárdenas 2015). En el contexto de la globalización del siglo XX, la modificación substancial del territorio desestimula el uso de las quemas, principalmente por los conflictos potenciales con los demás pobladores; análogo a otros pueblos originarios de sabanas, como los Krahô en Brasil (Mistry et al. 2005) y los Pemon en Venezuela (Rodríguez et al. 2016), incluso en otro tipo de ecosistemas como los forestales, donde las perturbaciones sobre el bosque se encuentran en el pensamiento de los adultos mayores del pueblo Anishinaabe (Ojibway) en el noroeste de Norte América (Miller y Davidson-Hunt 2010). Según Peñuela et al. (2011), la extensión de tenencia de la tierra se ha reducido en la zona y como consecuencia se ejerce mayor presión sobre al ambiente. Por consiguiente, en la actualidad, se tergiversa la importancia del fuego como configurador del paisaje llanero al evitar el manejo de las quemas de sabana y se atenúa el proceso de aculturación de los Sáliva. Esto concuerda con observaciones de Rodríguez et al. (2016) en sabanas de Venezuela, donde el uso del fuego en comunidades indígenas ha comenzado a evolucionar de un estado de latencia hacia un estado de conflicto manifiesto, en el que ha aumentado la conciencia pública sobre sus causas y ha comenzado a producirse un importante intercambio de perspectivas y saberes.

Bajo tales circunstancias, valorar y entender el saber tradicional que tienen los Sáliva sobre el papel y los efectos del fuego en las sabanas, coadyuvado con el saber del llanero raizal, resultaría útil para orientar planes de conservación del paisaje llanero y programas de desarrollo sostenible con pastizales nativos, mediante el uso racional de la quema. Ellos conocen el régimen de lluvias y la fisiografía de la sabana para determinar la relación período-lugar para quemar. Este es un conocimiento acertado para controlar recurrentes incendios que ocasionan desastres ambientales, y subsecuentemente mitigar presiones económicas y políticas sobre el uso intensivo del territorio que afectan tanto su entorno ecológico como cultural. La reflexión comunitaria sobre las dimensiones culturales de los conflictos ambientales puede permitir que se abran nuevos espacios de reflexión y discusión, que facilita el desarrollo de nuevas formas de gobernanza ambiental más justas en términos sociales y más consistentes en términos ambientales (Rodríguez et al. 2016). No obstante, la incidencia internacional por el cambio climático y los compromisos adquiridos por Colombia sobre reducción de gases de efecto invernadero son controversiales respecto a las quemas y a la ganadería. Acorde con Rodríguez et al. (2016), las quemas se relacionan con aspectos globales a tal punto de que la reducción de las emisiones del efecto invernadero proveniente de las quemas en ecosistemas de bosque y sabanas es un tema central de la agenda del Cambio Global. El tema del uso del fuego está dominado por valores, intereses y puntos de vista conflictivos que no facilitan para nada la tarea de lograr acuerdos sobre su uso sustentable. Para ello, es necesario comprender el conocimiento y la práctica de los pueblos originarios y redireccionar las percepciones eurocentristas sobre el uso tradicional de indígenas (Lewis 1989; Rodríguez 2007). Estigmatizar el uso del fuego en las sabanas no es la forma eficaz y de concientización sobre los impactos ambientales negativos de la quema, pues los Sáliva y la mayoría de ganaderos "blancos" mantienen la percepción de su necesidad; debe ser un proceso 
gradual de desmonte, facilitando y comprobando los beneficios de las prácticas sustitutivas.

\section{Conclusiones}

Por su característica sedentaria y concentración en resguardos, los Sáliva establecieron un pueblo disperso, lo que cambió la función básica del fuego hacia proteger sus viviendas de incendios; inicialmente tenían poco ganado, pero con la irrupción del "blanco" (p.ej., hatos ganaderos e industria petrolera) tuvieron presiones sobre su territorio que fueron compensadas con la donación de ganado y cercas de alambre, entre otras. Colateralmente los grandes hatos se fueron dividiendo y las nuevas fincas delimitadas con estas cercas. Esto obligó a Sálivas y ganaderos a aplicar quemas controladas para evitar conflictos con los vecinos; ambas comunidades perciben pocas alternativas de bajo costo y aplicables al ecosistema de sabana, para dejar de aplicar quema. Las razones principales para quemar la sabana son: obtener rebrote de pastizales frescos para la alimentación de bovinos con el propósito de aprovechar el consumo de varias especies de gramíneas que se lignifican rápidamente; impedir que se acumule el material vegetal seco que puede generar incendios incontrolables; disminuir insectos plagas, como zancudos, mosquitos y garrapatas, así como ahuyentar serpientes venenosas. En general, los Sáliva perciben más beneficios que perjuicios por uso del fuego; algunos prejuicios son el resultado de un atenuado proceso de aculturación, estrechamente relacionados con la imposición hipérbole del eurocentrismo sobre su territorio durante los siglos XIX, XX y XXI, que tergiversa la importancia del uso milenario del fuego en el paisaje de sabana inundable. Los resguardos indígenas, la reducción del tamaño de las fincas y la construcción de cercas han tenido impacto evidente en disminución y manejo controlado del fuego, hasta el punto de que algunos ya no lo aplican. Los pro y contras del uso del fuego no ameritan atribuir un carácter perverso o resaltar su aplicación, pero es indispensable profundizar su racionalidad y protección de nichos ambientalmente estratégicos y sensibles a severas afectaciones, como morichales, matas de monte y bosques de galería, mediante planificación predial y acuerdos de conservación/producción.

Agradecimientos: Este trabajo fue posible gracias a la colaboración del Grupo Orinoco de la Pontificia Universidad Javeriana. Grato agradecimiento a los pobladores Sálivas de los resguardos El Duya, San Juanito y Paravare. A la señora María Lucila Reyes, Presidente del Comité Departamental de Ganaderos de Casanare por su apoyo incondicional. Agradecemos a los tres revisores anónimos de Chungara Revista de Antropología Chilena, por sus valiosos comentarios y sugerencias que ayudaron a mejorar significativamente este manuscrito.

\section{Referencias Citadas}

Aedo, J.A. 2004. Conocimientos Ecológicos Locales y Desarrollo: el Caso de los Huicholes (México). Tesis para optar el grado de Maestría en Antropología y Desarrollo, Universidad de Chile, Santiago.

Cárdenas, I. 2015. Aproximación a los Conflictos Ambientales por Temas de Explotación de Hidrocarburos en los Resguardos del Pueblo Sáliba en Orocué, Casanare. Instituto de Investigación de Recursos Biológicos Alexander von Humboldt, Bogotá.

Fairhead, J. y M. Leach 1996. Misreading the African Landscape. Society and Ecology in a Forest-Savanna Mosaic. Cambridge University Press, Cambridge.

Fischer, J., D.J. Abson, V. Butsic, M.J. Chappell, J. Ekroos, J. Hanspach, T. Kuemmerle, H.G. Smith y H. von Wehrden 2014. Land sparing versus land sharing: Moving forward. Conservation Letters 7:149-157.

Huertas Herrera, A. 2006. Respuesta de la Estructura y Composición de la Vegetación en Tres Escenarios de Sabana Inundables Sometidos a Fuego durante la Etapa Temprana de Sucesión Municipio de Orocué, Casanare. Tesis para optar al grado de Ecólogo, Pontificia Universidad Javeriana, Bogotá.

Huertas Ramírez, H. 1977. Digestibilidad in Vitro de las Gramíneas Nativas Axonopus purpusii y Trachypogon vestitus en Suelo de Sabana de los Llanos Orientales. Tesis para optar el grado de Maestría, Universidad Nacional - ICA, Bogotá.

Huertas Ramírez, H. y A. Huertas Herrera 2015. Historiografía de la ganadería en la Orinoquia. Actas Iberoamericanas de Conservación Animal 6:300-307.

Iriarte, A. 2002. Impacto de la fauna silvestre sobre la flora nativa de Chile. En Agentes de Daño en el Bosque Nativo, editado por A. Baldini y L. Pancel, pp. 319-350. Editorial Universitaria, Santiago.

Jones, J. y J. Smith 2017. Ethnography: challenges and opportunities. Evidence Based Nursing 20:98-100.

Klaiber, J. 2007. Los Jesuitas en América Latina, 1549-2000: 450 Años de Inculturación, Defensa de los Derechos Humanos y Testimonio Profético. Fondo Editorial Universidad Antonio Ruiz de Montoya, Lima.

Laris, P. 2002. Burning the seasonal mosaic: preventative burning strategies in the wooded savanna of southern Mali. Human Ecology 30:155-186.

Lewis, H. 1989. Ecological and technical knowledge of fire: aborigines versus park rangers in northern Australia. American Anthropologist 91:940-961. 
Lindenmayer, D., R.J. Hobbs, R. Montague-Drake, J. Alexandra, A. Bennett, M. Burgman, P. Cale, A. Calhoun, V. Cramer, P. Cullen, D. Driscoll, L. Fahrig, J. Fischer, J Franklin, Y. Haila, M. Hunter, P. Gibbons, S. Lake, G. Luck, C. MacGregor, S. McIntyre, R. Mac Nally, A. Manning, J. Miller, H. Mooney, R. Noss, H. Possingham, D. Saunders, F. Schmiegelow, M. Scott, D. Simberloff, T. Sisk, G. Tabor, B Walker, J. Wiens, J. Woinarski y E. Zavaleta 2008. A checklist for ecological management of landscapes for conservation. Ecology Letters 11:78-91.

Mbow, C., T. Nielsen y K. Rasmussen 2000. Savanna fires in east-central Senegal: distribution patterns, resource management and perceptions. Human Ecology 28:561-583.

McGregor, S., V. Lawson, M. Christophersen, R. Kenneth, J. Boyden, P. Bayliss, A. Liedloff, B. McKaige y A. Andersen 2010. Indigenous Wetland Burning: conserving natural and cultural resources in Australia's World Heritage-listed Kakadu National Park. Human Ecology 38:721-729.

Miles, M.B. y A.M. Huberman 1994. Qualitative Data Analysis: An Expanded Sourcebook. SAGE Publications, INC., Thousand Oaks-London-New Delhi.

Miller, A.M. e I. Davidson-Hunt 2010. Fire, agency and scale in the creation of aboriginal cultural landscapes. Human Ecology 38:401-414.

Mistry, J., A. Berardu, V. Andrade, T. Krakô y O. Leonardos 2005. Indigenous fire management in the cerrado of Brasil The case of the Krakô of Tocantís. Human Ecology 33:365386.

Morey, R.V. 1972. Notes on the Sáliva of Eastern Colombia Current Anthropology 13:144-147.
Peñuela, L., A.P. Fernández, F. Castro y A. Ocampo 2011. Uso y Manejo de Forrajes Nativos en la Sabana Inundable de la Orinoquia. The Nature Conservancy - Fundación Horizonte Verde, Bogotá

Piraban, A.L. 2011. Diversidad Cultural del Departamento del Casanare. En Biodiversidad del Departamento de Casanare, Identificación de Ecosistemas Estratégicos, editado por J.S Usma y F. Trujillo, pp. 207-211. Gobernación de CasanareWWF Colombia, Bogotá.

Quintanilla, V. 2008. Perturbaciones a la vegetación nativa por grandes fuegos de 50 años atrás, en bosques Nordpatagónicos. Caso de estudio en Chile Meridional. Anales de Geografía 28:85-104

Rippstein, G., E. Amézquita, G. Escobar y C. Grollier 2001. Condiciones naturales de la sabana. En Agroecología y Biodiversidad de las Sabanas en los Llanos Orientales de Colombia, editado por G. Rippstein, G. Escobar y F. Motta, pp. 1-19. Centro Internacional de Agricultura Tropical, Cali.

Rodríguez, I. 2004. Conocimiento indígena vs científico: el conflicto por el uso del fuego en el Parque Nacional Canaima, Venezuela. Interciencia 29:121-129.

Rodríguez, I. 2007. Pemon perspectives of fire management in Canaima National Park, Venezuela. Human Ecology 35:331-343.

Rodríguez, I., B. Sletto, A. Leal, B. Bilbao e I. Sánchez-Rose 2016. A propósito del fuego: diálogo de saberes y justicia cognitiva en territorios indígenas culturalmente frágiles. TRILOGÍA. Ciencia, Tecnología y Sociedad 8:97-118.

Whelan, R. 1995. The Ecology of Fire. Cambridge University Press, Cambridge. 